\title{
Healthy middle-aged Asian and Caucasian populations present with large intra- and inter-individual variations of lower limb torsion
}

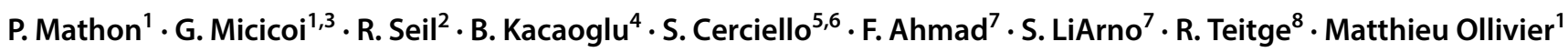

\begin{abstract}
Purpose There is a lack of standardization in the measurement of lower limb torsional alignment. Normal values published in the literature are inconsistent. A 3D-CT-scan-based method was used in a healthy population to define the femoral neck version (FNV) and the tibial torsion (TT) and their relationship with demographic parameters. The study objectives were (1) to define normal values of lower limb torsional alignment, (2) to estimate inter- and intra-individual variations of torsional deformity of healthy individuals' lower limbs. The hypothesis was that FNV and TT values would be influenced by patient characteristics such as gender, age, and ethnicity, and would have low side-to-side asymmetry.

Methods Torsional landmarks of the lower limbs from 191 healthy subjects were automatically calculated with a 3D CTscan-based program. The FNV was defined by the angle between the femoral neck axis and the femoral posterior condylar line. The TT angle was considered between the tibial plateau axis and the axis of the ankle. For the former, two alternatives were considered: the line connecting the more medial and lateral point of the medial and lateral plateau, respectively (method 1; TT1), or the line connecting the two more posterior points of the medial et lateral plateau (method 2; TT2). The ankle axis was defined as the line connecting the medial and lateral malleoli. These reference lines were automatically calculated. Age, gender, ethnic group, and BMI were recorded for every subject. A $p$ value $<0.05$ was considered as statistically significant. Results Overall, the mean FNV was $15.3 \pm 9.5^{\circ}$ and the mean TT was $31.6 \pm 6.3^{\circ}$. Female hips were more anteverted than male hips. Caucasians had less anteverted hips than Asians, but more externally rotated tibias. Age and BMI were not correlated with any anatomical parameter. A substantial side-to-side asymmetry was found for FNV [absolute difference $(\mathrm{AD})=6.3^{\circ}$; percentage of asymmetry $\left.(\% \mathrm{As})=47 \%\right]$, TT1 $\left(\mathrm{AD}=3^{\circ} ; \% \mathrm{As}=12 \%\right)$, and $\mathrm{TT} 2\left(\mathrm{AD}=4.9^{\circ} ; \% \mathrm{As}=9 \%\right)$ $(p=0.008)$.

Conclusion The findings showed that lower limb torsional parameters were highly variable from patient to patient and from one leg to the other for the same patient. The understanding of normal values concerning femoral version and external tibial torsion in the present healthy population will help surgeons to define pathological values of FNV and TT, as well as corrections to perform in case of torsional deformities.
\end{abstract}

Level of evidence Level III.

Keywords Lower limb torsion · Torsional alignment $\cdot$ Tibial torsion · Femoral neck version · Anteversion

\section{Introduction}

The lower limb presents a complex rotational profile; its skeleton has many twists around the longitudinal axis from the proximal end of the femur to the distal end of the tibia $[7,8]$.

Torsions have to be measurable to compare their functional effect on the hip, the knee, and the ankle. The study of the variations of angulation between those three joints should be the first step into the measurement of femoral and tibial torsions [2]. Concerning the normal femoral 
anteversion, studies have found similar results with $14 \pm 7^{\circ}$ in average, whereas a wide range of values from $20^{\circ}$ to $60^{\circ}$ have been found for the normal tibial torsion $[16,18,33]$.

Most of these results have been obtained from anatomical studies or two-dimensional CT measurements [21, 25, $27,30,35]$. The latter is the current gold standard for the measurement of torsional angles on the lower limb [36]. Yet, a large amount of methods has been described, but none of them has been shown to be superior to another. So far, all of them have failed in defining reproducible reference axis explaining the large range of normal torsional values in the femur and the tibia [34]. Derotation osteotomies are well described and efficient procedures when performed in patients with considerable knee symptoms associated with evidence of abnormal femoral version and/or tibial torsion $[15,29]$. However, a clear evaluation using a modern 3D morphological of normal values (range) in healthy individuals is needed to define torsional abnormalities cut-off.

The objectives of the current study were (1) to define normal values of lower limb torsion, and (2) to estimate inter- and intra-individual variations of torsional alignment of healthy individuals' lower limbs. The hypothesis was that FNV and TT values would be influenced by patient characteristics such as gender, age and ethnicity, and would present with a low side-to-side asymmetry.

\section{Material and methods}

After local ethic committee approval (Aix-Marseille University, CIL no. 2018-12-234), the CT scans of the pelvis and bilateral femur of 191 normal individuals were retrieved from the SOMA database (Stryker, Mahwah, New Jersey) $[1,6,28]$.

An automatic CT-scan-based modeling and analytic system was used for this study. The pelvis and bilateral femur of 191 patients (90 males/101 females, mean age $59.8 \pm 15$ years (18-84 years) and mean BMI $24.7 \pm 4.8 \mathrm{~kg} /$ $\mathrm{m}^{2}$ ) including two ethnicities (93 Asian and 98 Caucasian) were examined. Subjects with surgical hardware, bony deformities, and osteoarthritic joints were excluded, because this could have modified parameters of interest. The demographic parameters evaluated in the analysis included age, gender, height, weight, body mass index, and ethnicity. Each of the measurements were made with algorithm-based constructions in an index bone. Then, these measurements were mapped to each bone of the database by a previously validated, automated software (Soma TM, Stryker, Mahwah, US), resulting in reproducible and consistent parameters for each subject $[1,6,28]$. The femoral neck version (FNV) and the tibial torsion (TT) were calculated. Inter-observer reliability was not estimated as all parameters were automatically extracted from the software. Intra-observer reliability was assessed using two independent extractions; the intraclass correlation coefficient was rated excellent (>99\%). The precision of the automated extraction system used in this study protocol was published to be associated with a margin of error of less than $2 \mathrm{~mm}$ and less than $1^{\circ}$ (which is related to the CT slicing process) $[1,6,28]$ (Fig. 1).

The femoral neck axis and the anatomical axis of the femur were first selected from the pre-defined landmarks available. These axes were then projected onto the frontal plane and the angle between both axes determined [26]. Next, the most posterior points on the medial and lateral condyle were chosen from the pre-defined landmark list and the points utilized to define the posterior condylar axis (PCA). The PCA and the femoral neck axis, chosen above, were projected onto the transverse plane and the angle between them measured as the femoral neck version (Fig. 1).

To define tibial torsion, two methods were used to define the tibial proximal axis using either the line connecting the more medial and lateral point of the medial and lateral plateau, respectively, method 1 (TT1) or the line connecting the two more posterior points of the medial et lateral plateau-method 2 (TT2) and the ankle axis (defined as the line connecting the medial and lateral malleoli) and an axis established between them in the axial plane (Fig. 2).

For the distal axis, the following method was used, the medial malleolus was selected as a pre-defined landmark, and the lateral malleolus was established by projecting a point on the most lateral aspect of the fibula [26]. An axis was drawn between the two malleoli and projected onto the axial plane. The angle between the proximal tibial axis and the malleolar axis was used to define the tibial torsion for each subject. Previous evaluation of intra and inter-observer reproducibility of this method has been estimated to provide

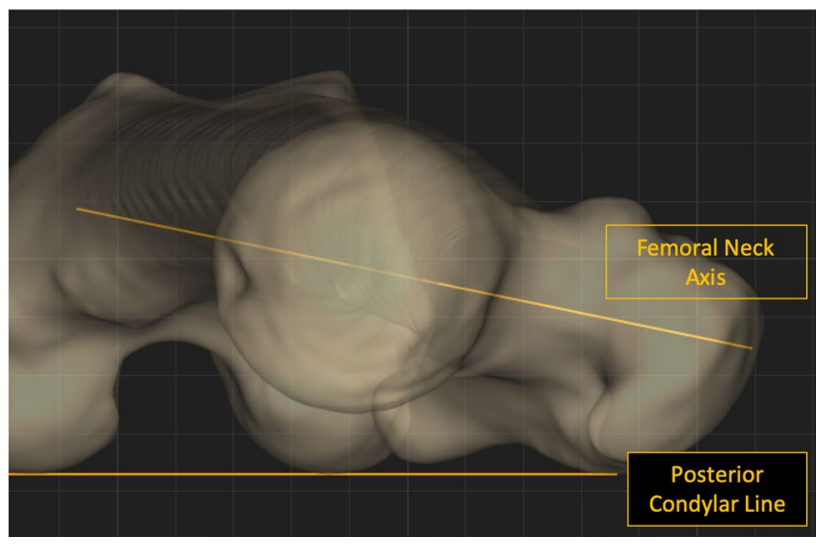

Fig. 1 Femoral version measurement. The most posterior points on the medial and lateral condyle were chosen from the pre-defined landmark list and the points utilized to define the posterior condylar axis (PCA). The PCA and the femoral neck axis were projected onto the transverse plane and the angle between them measured as the femoral neck version 


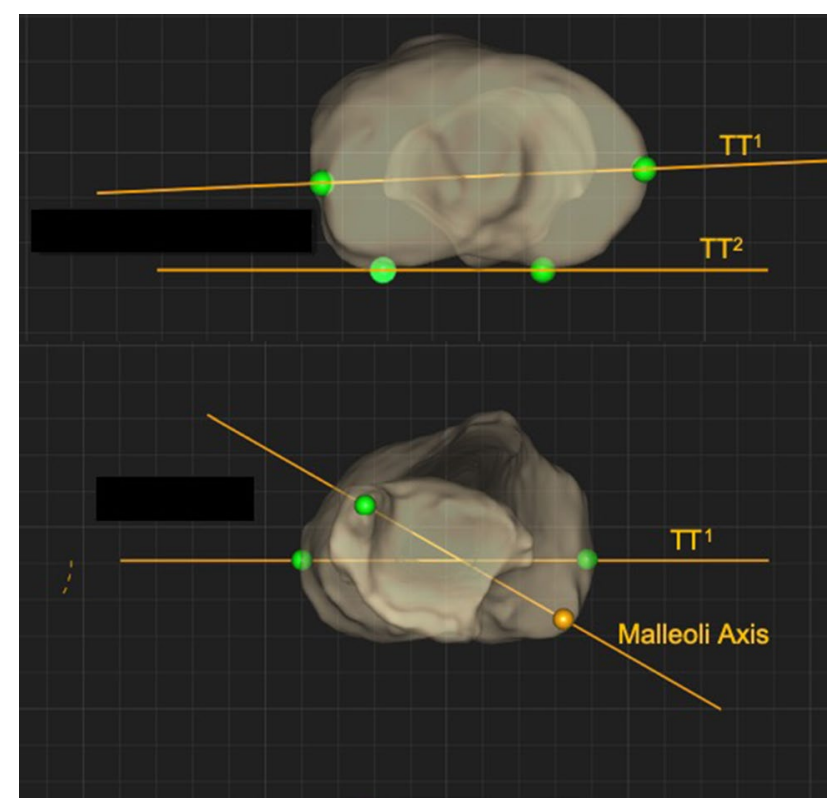

Fig. 2 Tibial torsion measurement. Two methods were used to define the tibial proximal axis using either the line connecting the more medial and lateral point of the medial and lateral plateau respectively method 1 (TT1) or the line connecting the two more posterior points of the medial et lateral plateau-method 2 (TT2) and the ankle axis (defined as the line connecting the medial and lateral malleoli) and an axis established between them in the axial plane

results with a intra-class correlation coefficient $>0.9$ and a margin of error $<1^{\circ}$ or $<1 \mathrm{~mm}$ imputated to CT-scan slicing process [26].

\section{Statistical analysis}

Means and standard deviations were determined for each of the measurements made for the population as a whole and for subpopulations, based on gender, ethnicity, and age. Normal (Gaussian) distributions were determined. Univariate analysis was performed using $t$ tests to estimate a difference between groups and subgroups. Pearson's coefficients were calculated to examine correlations among the demographic data of the specimen and femoral measurements. Multiple linear regression models were developed to establish the determinants for each of the variables. For each model, variables with a $p$ value less than 0.1 were kept in the final model.

Side-to-side absolute differences (AD) were obtained by subtracting the result of the smaller of the specimens (left or right) from the result of the larger. Percent absolute asymmetry was determined by using the following documented method: percent asymmetry $=($ maximum - minimum)/(average of maximum and minimum). This variable was used to define the magnitude of asymmetry. A sideto-side difference $>2 \%$ was defined as "substantial asymmetry". The sample size was defined based on the expected $\mathrm{FNV}=14.5 \pm 8.1$ [17] for a required level of statistical significance of $\alpha=0.05$, and a power of $1-\beta=0.80$. One hundred and twelve lower limbs would be required to detect a difference $>2^{\circ}$ between groups and subgroups. A trained statistician (MO) performed statistical analysis using SPSS software (Version 22; SPSS Inc, Chicago, IL, USA). All calculations will assume two-tailed tests.

\section{Results}

Overall, the mean FNV was $15.3 \pm 9.5^{\circ}\left(0^{\circ}-70^{\circ}\right)$. The mean TT1 was $31.6 \pm 6.3^{\circ}\left(14^{\circ}-48^{\circ}\right)$ and the mean TT2 was $34.6 \pm 7.0^{\circ}\left(15^{\circ}-53^{\circ}\right)$ (Fig. 3). Regarding the gender subgroup analysis, the mean FNV was $11.6 \pm 6.6^{\circ}$ and $18.5 \pm 10.5^{\circ}$ for males and females, respectively $(p<0.0001)$. The mean TT1 was $31.2 \pm 5.7^{\circ}$ and $31.9 \pm 6.7^{\circ}$ for males and females, respectively (n.s.). The mean TT2 was $34.3 \pm 6.4^{\circ}$ and $34.9 \pm 7.5^{\circ}$ for males and females, respectively (n.s.) (Table 1).

With respect to the ethnic subgroup, a mean FNV of $14.0 \pm 7.6^{\circ}$ for Caucasians and $16.7 \pm 11.3^{\circ}$ for Asians was

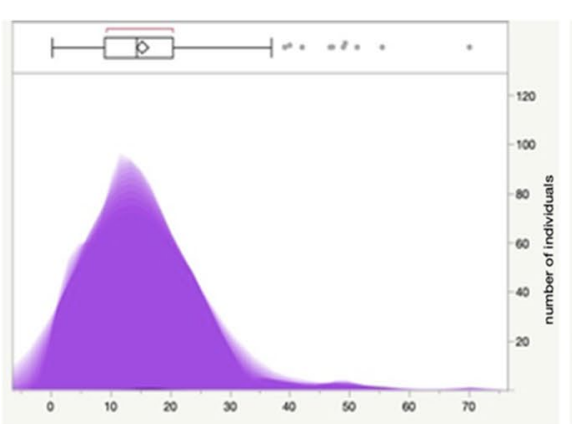

a

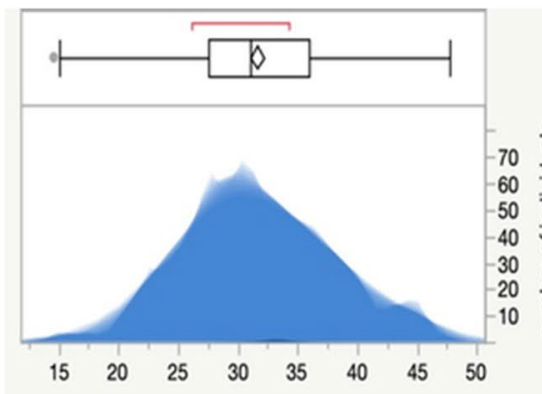

b

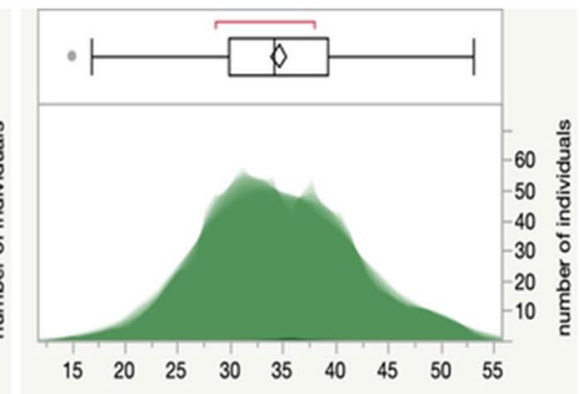

c

Fig. 3 Distribution of the series population. a Femoral version, b tibial torsion (method 1), and $\mathbf{c}$ tibial torsion (method 2) in the series population 
Table 1 Analysis of differences in anatomical parameters by gender, $p$ value calculated with Student's $t$ test

\begin{tabular}{|c|c|c|c|c|}
\hline $\begin{array}{l}\text { Anatomic } \\
\text { parameter } \\
\left({ }^{\circ}\right)\end{array}$ & Males (SD) & Females (SD) & $\begin{array}{l}\text { Difference (CI } \\
95 \%)\end{array}$ & $p$ value \\
\hline FNV & $11.6(6.6)$ & $18.5(10.5)$ & $\begin{array}{l}-6.9(-8.7 \\
-5.1)\end{array}$ & $<0.0001$ \\
\hline TT1 & $31.2(5.7)$ & $31.9(6.7)$ & $\begin{array}{l}-0.7[-1.9 \\
0.5]\end{array}$ & n.s \\
\hline TT2 & $34.3(6.4)$ & $34.9(7.5)$ & $-0.6[-2 ; 0.8]$ & n.s \\
\hline
\end{tabular}

$C I$ confidence interval, $S D$ standard deviation, $n . s$. not significant

measured $(p=0.007)$. The mean TT1 was $33 \pm 6.5^{\circ}$ and $30.1 \pm 5.7^{\circ}$ for Caucasians and Asians $(p<0.0001)$, respectively. The mean TT2 was $35.8 \pm 7.5^{\circ}$ for Caucasians and $33.4 \pm 6.3^{\circ}$ for Asians $(p=0.001)$ (Table 2 ).

No correlation was found in the multivariate analysis between age $(R=0.04$, n.s. $)$, BMI $(R=0.1$, n.s. $)$, and FNV. No correlation was found between age $(R=0.07$, n.s. $)$, BMI $(R=0.21$, n.s. $)$, gender $(R=0.08$, n.s. $)$, and TT1; as well as between age $(R=0.05$, n.s. $)$, BMI $(R=0.17$, n.s. $)$, gender $(R=0.09$, n.s. $)$, and TT2. A statistically significant relationship was found between FNV $(R=0.3, p=0.01)$, TT1 ( $R=0.5, p=0.008)$, TT2 $(R=0.5, p=0.009)$, and ethnicity.

The side-to-side analysis exhibited a significant asymmetry for FNV $\left(\mathrm{AD}=6.3^{\circ}, \% \mathrm{As}=47 \% ; p<0.0001\right)$, TT1 $\left(\mathrm{AD}=3^{\circ}, \% \mathrm{As}=12 \% ; p=0.007\right)$, and $\mathrm{TT} 2(\mathrm{AD}=4.9 \%$, $\% \mathrm{As}=9 \% ; p=0.008)$.

\section{Discussion}

The main interests of this study consisted in the definition of normal values of femoral and tibial torsion in a large group of patients presenting with various demographic parameters using a robust 3D CT-scan method. The identified torsional parameters yielded substantial inter-individual variations which were mostly related to gender and ethnicity, as well as intra-individual side-to-side variations.

Estimate the impact of torsion on lower limb unction entails defining the axis of motion of each joint and the angle or vector between those axes. As no method, to date, allows determination on CT (2D or 3D) of the exact motion axes, this study selects points on the proximal femur, distal femur, proximal tibia, and distal tibia to draw lines which were arbitrary defined as potential approximation of joint motion axes. Several methods have been described in attempt to define them [11, 20, 34, 37]. Two-dimensional CT-scan methods have been advocated to define those elements, but measuring an angle in two dimensions introduces an inherent error due to the approximation of a complex tri-dimensional structure into a two-dimensional structure [5]. As such, comparison of the results to the actual literature is difficult, methods used to define torsion deeply vary from study to study. The main difficulty consists in defining a reproducible femoral neck axis [11, 20, 34, 37]. Murphy et al. suggested taking the line joining the center of the femoral head and the center of the femoral shaft on a level just below the lesser trochanter [20]. However, this line does not always follow the center of the femoral neck which is often curved. Because of that, other authors suggested taking a line cutting in half the neck drawn along two points measured in the center of the neck $[11,37]$. Values of the FNV vary in a significant proportion depending on the type of measurement method.

The same situation exists in the tibia to define the proximal axis. Several reference axes have been arbitrarily defined on the tibial plateau, but depending on the CT slice chosen, the direction of the axis changes and so does the value of the tibial torsion $[4,24,39]$. Thus, there is no reliable technique for measuring the torsion of the lower limb. This lack of standardization leads to a wide range of normal torsional values and it is, therefore, difficult to compare datasets from one method to another [34].

In this series, a 3D CT-scan-based method was selected to measure FNV and TT. The use of 3D imaging allows to avoid selecting two 2D images to perform angle measurements which increase data deviation [20]. This 3D method automatically identifies landmarks with a best accuracy than manual mapping. The features of interest (axes) were marked on a template, and then, the system automatically extracted them from all selected database individuals. Previous validation of this method reported $\mathrm{a}<2 \mathrm{~mm}$ or $<2^{\circ}$ precision [6, 28]. The mean FNV in this study was $15.3 \pm 9.5^{\circ}$ as compared with the $8.1^{\circ}$ [14] to $24.1^{\circ}$ [30] range published in the literature. This mean value is in accordance with two other studies having measured the anatomic characteristics of the proximal femur based on $3 \mathrm{D}$ reconstructions $[3,10]$. The
Table 2 Analysis of differences in anatomical parameters by ethnicity, $p$ value calculated with Student's $t$ test

\begin{tabular}{llllr}
\hline $\begin{array}{l}\text { Anatomical param- } \\
\text { eters }\left(^{\circ}\right)\end{array}$ & Caucasians $(\mathrm{SD})$ & Asians (SD) & Difference (CI 95\%) & $p$ value \\
\hline FNV & $14(7.6)$ & $16.7(11.3)$ & $-2.6(0.8 ; 4.5)$ & 0.007 \\
$\mathrm{TT}^{1}$ & $33(6.5)$ & $30.1(5.7)$ & $2.8(1.6 ; 4.1)$ & $<0.0001$ \\
$\mathrm{TT}^{2}$ & $35.8(7.5)$ & $33.4(6.3)$ & $2.4(1 ; 3.7)$ & 0.001 \\
\hline
\end{tabular}

$C I$ confidence interval, $S D$ standard deviation 
range of values published in the literature for the TT is even wider than for the FNV with mean values spreading from $20^{\circ}$ to $41.7^{\circ}[13,27]$. When the centers of the tibial plateau were defined as proximal axis, a mean value of $31.6 \pm 6.3^{\circ}$ was identified in the present study. When the tibial posterior condylar line was chosen, a mean value of $34.6 \pm 7^{\circ}$ was found. This result is close to the findings of Liodakis et al. and Waidelich et al. (respectively, $28.5^{\circ}$ and $33.1^{\circ}$ ) which are both studies based on 2D CT-scan imaging [19, 35].

To the best of the authors' knowledge, there is no study having analyzed the tibial torsion from $3 \mathrm{D}$ reconstructions. It is, therefore, impossible to compare the results of this study because of the heterogeneity of the measurement methods used in the literature. Concerning the subgroup analysis, a higher FNV $\left(6.9^{\circ}\right)$ was found in female subjects. Carmona et al. [3] also found that women had a significantly superior FNV but, to a reduced extent, with a mean $2.2^{\circ}$ difference. Ethnicity also influences FNV values, with Asians having a more anteverted femoral neck than Caucasians $\left(2.6^{\circ}\right)$. This difference had been found previously by Hartel et al. [10]. The mean values of FNV in Asians of the present study are close to those found by Zhang and Park who studied a Chinese population $\left(16.4^{\circ}\right.$ and $17.3^{\circ}$, respectively) [23, 38]. The TT was also significantly lower in A sian subjects with a mean difference of $2.8^{\circ}[8,12,31,32]$. Those results are consistent with a study from Hovinga and Lerner in limited samples of 23 Japanese and 47 Caucasian subjects [12]. The authors described lower TT in the Japanese than in the Caucasian group (mean difference $5 \pm 10^{\circ}$ ). The studies of Tamara et al. investigated femoral and tibial torsions in a Japanese and Caucasian/Australian population $[31,32]$. They failed to demonstrate morphological differences between groups, but acknowledged that osteoarthritis changes in the knees of their subjects might have decreased the precision of their analysis which was not based on 3D imaging. No comparison of the TT based on ethnicity, gender, age, or BMI could be identified in the literature.

A significant asymmetry was found between the left side and the right side for both femoral and tibial torsion. This result is in line with two 3D studies on the proximal femur $[3,10]$. Other studies have found differences side-to-side difference between TT values based using 2D measurement [30, 34].

It is known that long bones of the lower limbs modify their torsion during growth [8]. Little is known about changes occurring during later life. In the present study, no relation between age and femoral or tibial torsion could be identified. One may assume that the reason for this was the exclusive inclusion of subjects over the age of 18 years. However, some studies managed to show the impact of age on FNV [9, 10]. Finally, BMI was not a factor impacting the FNV or the TT which confirms the results of previous studies [3, 10, 22].
This study has several limitations. First, individual characteristics such as activity level or dominance, which have the potential to impact the torsion of the femur and the tibia, could not be considered because of the retrospective design of the study. The population of this study may not be comparable to patients which are eligible for hip or knee surgeries. Finally, the measurements were limited to Caucasian and Asian subjects only and data focusing on other ethnicities are needed to define torsional normality. Finally, this study only approximated torsion using previously but arbitrary defined landmarks, following a robust but complex method, defining points, lines, and angles with an automated software. As such, measurement depicted in this research project was obtained using a specific process that might have influenced morphological outcomes. Using the same landmarks but a different software, dataset and study protocol other authors could find substantially different results.

This study determined normal values of lower limb torsion, using a robust 3D-CT analysis. For patients with considerable knees symptoms and based on this study reported morphometric data, cut-off values for abnormal torsion (outside of the $95 \%$ confidence interval FNV $>35^{\circ}$ and/or $\mathrm{TT}>43^{\circ}$ ) might help surgeons to decide and to plan correction of those morphometric abnormalities.

\section{Conclusion}

The findings showed that lower limb torsional parameters were highly variable from patient to patient and from one leg to the other for the same patient. The understanding of normal values concerning femoral version and external tibial torsion in the present healthy population will help surgeons to define pathological values of FNV and TT, as well as corrections to perform in case of torsional deformities.

Author contributions SL and AF performed data extraction, MO, RS, and RT designed the protocol. MO performed statistical analysis. PM and GM wrote the initial draft, and SC and BK edited the different versions of the draft. PM, GM, RS, RT, SC, BK, AF, SL, and MO approved the submitted and final versions.

Funding No funding was needed for this study.

\section{Compliance with ethical standards}

Conflict of interest PM, GM, SC, BK, and RT have nothing to disclose. MO is an educational consultant for Stryker, New-clip and Arthrex. SL and AF are paid employee of stryker. RS is board member of ESSKA, and Consultant for Smith and Nephew.

Ethical approval The local ethics committee approved our study protocol prior to investigation. 


\section{References}

1. Banerjee S, Faizan A, Nevelos J, Kreuzer S, Burgkart R, Harwin SF, Mont MA (2014) Innovations in hip arthroplasty threedimensional modeling and analytical technology (SOMA). Surg Technol Int 24:288-294

2. Bouchard R, Meeder PJ, Krug F, Libicher M (2004) Evaluation of tibial torsion - comparison of clinicalmethods and computed tomography. Rofo 176:1278-1284

3. Carmona M, Tzioup C, LiArno S, Faizan H, Argenson JN, Ollivier M (2019) Upper femur anatomy depends on age and gender: a three-dimensional computed tomography comparative bone morphometric analysis of 628 healthy patients. Hips J Arthroplast $34: 2487-2493$

4. Cobb JP, Dixon H, Dandachli W, Iranpour F (2008) The anatomical tibial axis. J Bone Jt Surg Br 90-B(8):1032-1038

5. Dargel J, Feiser J, Gotter M, Pennig D, Koebke J (2009) Side differences in the anatomy of human knee joints. Knee Surg Sports Traumatol Arthrosc 17:1368-1376

6. Gottschling H, Schröder M, Reimers N, Fischer F, Homeier A, Burgkart R (2009) A system for performing automated measurements on large bone databases. IFMBE Proc 25:910-913

7. Grisch D, Dreher T (2019) Torsion and torsional development of the lower extremities. Orthopade 48:523-530

8. Guidera KJ, Ganey TM, Keneally CR, Ogden JA (1994) The embryologyoflower-extremity torsion. Clin Orthop Relat Res 302:17-21

9. Gulan G, Matovinović D, Nemec B, Rubinić D, Ravlić-Gulan J (2000) Femoral neck anteversion: values, development, measurement, common problems. Coll Antropol 24:521-527

10. Hartel MJ, Petersik A, Schmidt A, Kendoff D, Nüchtern J, Rueger JM, Lehmann W, Grossterlinden LG (2016) Determination of femoral neck angle and torsion angle utilizing a novel threedimensional modeling and analytical technology based on CT datasets. PLoS ONE 11(3):e0149480. https://doi.org/10.1371/ journal.pone. 0149480

11. Hernandez RJ, Tachdjian MO, Poznanski AK, Dias LS (1981) CT determination of femoral torsion. Am J Roentgenol 137:97-101

12. Hovinga KR, Lerner AL (2009) Anatomic variations between Japanese and Caucasian populations in the healthy young adult knee joint. J Orthop Res 27:1191-1196

13. Hutter CG, Scott W (1949) Tibial torsion. J Bone Jt Surg 31A:511-518

14. Jain AK, Maheshwari AV, Nath S, Singh MPNM (2003) Anteversion of the femoral neck in Indian dry femora. J Orthop Sci 8:334-340

15. Kaiser P, Konschake M, Loth F, Plaikner M, Attal R, Liebensteiner M, Schlumberger M (2020) Derotational femoral osteotomy changes patella tilt, patella engagement and tibial tuberosity trochlear groove distance. Knee Surg Sports Traumatol Arthrosc 28:926-933

16. Kinzinger H, Castiaux P (1977) Les vices de torsion des membres inférieurs. Historique, évolution clinique. Acta Orthop Belg 43:379-470

17. Laumonerie $\mathrm{P}$, Cavaignac E, Ollivier M, Argenson JN, LiArno S, Faizan A (2018) Which factors influence proximal femoral asymmetry? Bone Jt J 100B:839-844

18. Lerat JL (1982) Taussig G Les anomalies de rotation des membres inférieurs. Rev Chir Orthop 68:1-74

19. Liodakis E, Doxastaki I, Chu K (2012) Reliability of the assessment of lower limb torsion using computed tomography: analysis of five different techniques. Skelet Radiol 41:305-311

20. Murphy SB, Simon SR, Kijewski PK, Wilkinson RH, Griscom NT (1987) Femoral anteversion. J Bone Jt Surg 69:1169-1176
21. Noble PC, Alexander JW, Lindahl LJ (1988) The anatomic basis of femoral component design. Clin Orthop Relat Res 235(6):148-165

22. Ollivier M, Parratte S, Lecorroller T, Reggiori A, Champsaur P, Argenson J-N (2014) Anatomy of the proximal femur at the time of total hip arthroplasty is a matter of morphotype and etiology but not gender. Surg Radiol Anat 4:377-384

23. Park J, Kim JY, Kim HD, Kim YC, Seo A, Je M, Mun UJ, Bia K, Park HI, Kim SY (2017) Analysis of acetabular orientation and femoral anteversion using images of three-dimensional reconstructed bone models. Int J Comput Assist Radiol Surg 12(10):855-864

24. Reikerås O, Høiseth $\mathrm{A}$ (1989) Torsion of the leg determined by computed tomography. Acta Orthop Scand 60:330-333

25. Rubin PJ, Leyvraz PF, Aubaniac JM (1992) The morphology of the proximal femur. A three-dimensional radiographic analysis. $\mathrm{J}$ Bone Jt Surg 74:28-32

26. Schmidt W, LiArno S, Khlopas A, Petersik A, Mont MA (2018) Stryker orthopaedic modeling and analytics (SOMA): a review. Surg Technol Int 32:315-324

27. Schneider B, Laubenberger J, Jemlich S, Groene K, Weber HM, Langer M (1997) Measurement of femoral antetorsion and tibial torsion by magnetic resonance imaging. Br J Radiol 70:575-579

28. Schröder M, Gottschling H, Reimers N, Hauschild M, Burgkart R (2014) Automated morphometric analysis of the femur on large anatomical databases with highly accurate correspondence detection. Open Med J 1:15-22

29. Schröter S, Nakayama H, Ihle C, Ateschrang A, Maiotti M, Harrer J, Dickschas J (2019) Torsional osteotomy. J Knee Surg. https:// doi.org/10.1055/s-0039-1678677

30. Strecker W, Keppler P, Gebhard F, Kinzl L (1997) Length and torsion of the lower limb. J Bone Jt Surg 79:1019-1023

31. Tamari K, Briffa K, Tinley P, Aoyagi K (2007) Variations in torsion of the lower limb in Japanese and Caucasians with and without knee osteoarthritis. J Rheumatol 34:145-150

32. Tamari K, Tinley P, Briffa K, Aoyagi K (2005) Ethnic-, gender-, and age-related differences in femorotibial angle, femoral antetorsion, and tibiofibular torsion: cross-sectional study among healthy Japanese and Australian Caucasians. Clin Anat 19:59-67

33. Taussig G (1995) Les anomalies de rotation des membres inférieurs chez l'enfant. J de Pediatrie et Pueric 8:131-136

34. Vanhove F, Noppe N, Fragomen A, Hoekstra H, Vanderschueren G, Metsemakers WJ (2019) Standardization of torsional CT measurements of the lower limbs with threshold values for corrective osteotomy. Arch Orthop Trauma Surg 139:795-805

35. Waidelich H, Strecker W, Schneider E (1992) Computed tomographic torsion-angle and length measurement of the lower extremity. The methods, normal values and radiation load. Fortschr Röntgenstr 157:245-251

36. Waldt $S$ (2013) Lower limb alignments. In: Waldt S (ed) Measurements and classifications in musculoskeletal radiology. George Thieme Verlag KG, New York, pp 5-10

37. Weiner DS, Cook AJ, Hoyt WA Jr, Oravec CE (1978) Computed tomography in themeasurement of femoral anteversion. Orthopedics 1:299-306

38. Yang Z, Jian W, Zhi-Han L, Jun X, Liang Z, Ge Y, Zhan-Jun S (2014) The geometry of the bone structure associated with total hip arthroplasty. PLoS ONE 9(3):e91058. https://doi.org/10.1371/ journal.pone.0091058

39. Yoshioka Y, Siu DW, Scudamore RA, Cooke TDV (1989) Tibial anatomy and functional axes. J Orthop Res 7:132-137 


\section{Affiliations}

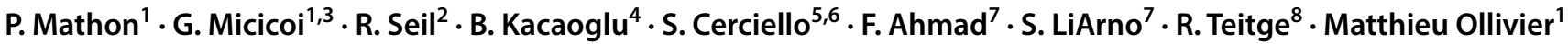

1 Department of Orthopedics and Traumatology, Institute of Movement and Locomotion, Sainte-Marguerite Hospital, Aix Marseille University, APHM, CNRS, ISM, 270 Boulevard Sainte Marguerite, BP 29, 13274 Marseille, France

2 Centre Hospitalier Universitaire du Luxembourg, Luxembourg, Luxembourg

3 iULS-University Institute for Locomotion and Sports, Pasteur 2 Hospital, University Côte D’Azur, Nice, France

4 Department of Orthopedic Surgery, Acibadem Altunizade Hospital, Acibadem University, Faculty of Medicine,
Tophanelioglu Cad. No: 1, Altunizade, Uskudar, Istanbul, Turkey

5 Marrelli Hospital, Crotone, Italy

6 Casa Di Cura Villa Betania, Rome, Italy

7 Stryker, Mawhaw, NJ, USA

8 Detroit Medical Center, 3990 John R St., Detroit, MI 48201, USA 